\title{
Wavelet Packets-Based Blind Watermarking for Medical Image Management
}

\author{
Salwa A.K. Mostafa ${ }^{*}$, Naser El- sheimy $^{2}$, A.S. Tolba ${ }^{3}$, F.M. Abdelkader ${ }^{1}$ and Hisham M. Elhindy ${ }^{1}$ \\ ${ }^{I}$ Department of Electrical Engineering, Faculty of Engineering, Suez-Canal University, Egypt \\ ${ }^{2}$ Department of Geomatics Engineering, Faculty of Engineering, University of Calgary, Calgary, Canada \\ ${ }^{3}$ Faculty of Computer Studies, Arab Open University, Kuwait, $H Q$
}

\begin{abstract}
The last decade has witnessed an explosive use of medical images and Electronics Patient Record (EPR) in the healthcare sector for facilitating the sharing of patient information and exchange between networked hospitals and healthcare centers. To guarantee the security, authenticity and management of medical images and information through storage and distribution, the watermarking techniques are growing to protect the medical healthcare information. This paper presents a technique for embedding the EPR information in the medical image to save storage space and transmission overheads and to guarantee security of the shared data. This paper presents a new method for protecting the patient information in which the information is embedded as a watermark in the discrete wavelet packet transform (DWPT) of the medical image using the hospital logo as a reference image. The patient information is coded by an error correcting code (ECC), $\mathrm{BCH}$ code, to enhance the robustness of the proposed method. The scheme is blind so that the EPR can be extracted from the medical image without the need of the original image. Therefore, this proposed technique is useful in telemedicine applications. Performance of the proposed method was tested using four modalities of medical images; MRA, MRI, Radiological, and CT. Experimental results showed no visible difference between the watermarked and the original image. Moreover, the proposed watermarking method is robust against a wide range of attacks such as JPEG coding, Gaussian noise addition, histogram equalization, gamma correction, contrast adjustment, and sharpen filter and rotation.
\end{abstract}

Keywords: Medical image watermarking (MIW), electronic patient record (EPR), discrete wavelet packet transform DWPT, $\mathrm{BCH}$ code.

\section{INTRODUCTION}

Recently, telemedicine applications in teleconsulting, telediagnoisis, telesurgery and remote medical education play a vital rule in the evolution of the healthcare domain [1]. The transmission, storage and sharing of electronic medical data via the networks have many purposes such as diagnosis, finding new drugs and scientific research. Hospitals and medical centers have huge databases including medical images, text and patient records. The exchange of these databases through the networks requires content management to index medical record information and a high degree of security and authenticity to preserve the privacy of the patients' information. To achieve these objectives, different techniques of digital watermarking have been employed. Ananad et al. [2] proposed a technique for watermark medical images with encrypted patient data in the spatial domain by swapping the lower significant bits of the gray values of chosen pixels of the medical image with that of the watermark. In [3], the encrypted patient information which is coded using error control codes is swapped with the least significant bit (LSB) of the grey scale image. Nambakhsh et al. [4] presented a watermarking method

\footnotetext{
*Address correspondence to this author at the Department of Electrical Engineering, Faculty of Engineering, Suez-Canal University, Egypt; Tel: +1 (403) 7648475; Fax: +1 (403) 4677324;
}

E-mail: abdosaad2002@hotmail.com combined with the embedded zero-tree wavelet algorithm (EZW). The watermark is embedded by replacing the significant wavelet coefficients of ECG signals by the corresponding significant wavelet coefficients of the host image. Aggeliki et al. [5] proposed a wavelet-based multiple watermarking scheme. In this scheme, four types of watermarks are embedded into the wavelet coefficients of medical images using a quantization of selected coefficients. Kallel et al. [6] developed an algorithm for embedding the patient's diagnosis in the spatial domain of medical image. A hash function is applied to 7 bit plane of image and XORed with the coded diagnosis inserted by the doctor. The resulting message is inserted in LSB plane. Huang et al. [7] implemented an algorithm which uses a part of patient's record in EXIF metadata as a watermark. The 8x8 blocks discrete cosine transform (DCT) is performed on the medical image and the middle frequency coefficients of each block are chosen to embed watermark bits. Most of previous research in literature has focused on preserving the resolution of the medical images after embedding the watermark regardless of testing the robustness of the schemes against different attacks.

Our research focuses on developing a watermarking algorithm to hide electronic patient record (EPR) in the medical image. The confidentiality of patient data is improved by hiding the EPR data as a watermark. In addition 
to that, both the storage and transmission bandwidth requirements for medical images are reduced.

The Digital Imaging and Communications in Medicine (DICOM) standard is the standard to exchange medical data. The DICOM medical image files are attached with header containing patient information which may be lost, attacked or disordered with other header file. However, the watermarking of medical images using patient information overcomes these problems [8]. However, there is a challenge that interleaving data in a medical image must not affect the image quality as this may result in wrong diagnosis.

In this paper, a new blind watermarking method is suggested. The medical image is transformed to two level wavelet packet transform. According to the energy of each band, we select two bands for watermarking to balance between robustness and impeccability. The watermark is the EPR which includes index information, patient information, diagnosis and some data related to the medical case. All the information is converted to binary form and coded by Error Correcting Code (ECC) to enhance robustness. A reference image selected as a binary logo of the medical center is used to embed the watermark. This paper is organized as follows; section 2 reviews the basic idea of DWPT. The proposed algorithm is described in Section 3. In section 4, the experimental results are introduced and discussed. Finally section 5 concludes the paper.

\section{DISCRETE WAVELET PACKET TRANSFORM}

The 2D- wavelet packet transform is a generalization of 2D- wavelet transform. It is a flexible tool offering richer image resolutions. In the orthogonal wavelet decomposition procedure, the generic step splits only the approximation coefficients sub-band of the image into four sub-bands. After this split, we obtain a sub-band of approximation coefficients (LL) and three sub-bands of detail coefficients (LH - HL $\mathrm{HH}$ ). The next step consists of splitting the new approximation coefficient sub-band in a recursive manner where the successive details sub-bands are never reanalyzed. In the corresponding wavelet packet situation, each detail coefficients sub-band is also decomposed into four sub-bands using the same approach as in approximation subband splitting. The DWPT for two levels is shown in Fig. (1).

The wavelet packet transform for an image gives a vast amount of sub-bands of wavelet coefficients at different resolutions. This allows more flexibility to select a sub-band or more to embed watermark and this will increase the watermarking security $[9,10]$.

\begin{tabular}{|c|c|c|c|}
\hline $\mathrm{LL}_{2} \mathrm{LL}_{1}$ & $\mathrm{HL}_{2} \mathrm{LL}_{1}$ & $\mathrm{LL}_{2} \mathrm{HL}_{1}$ & $\mathrm{HL}_{2} \mathrm{HL}_{1}$ \\
\hline $\mathrm{LH}_{2} \mathrm{LL}_{1}$ & $\mathrm{HH}_{2} \mathrm{LL}_{1}$ & $\mathrm{LH}_{2} \mathrm{HL}_{1}$ & $\mathrm{HH}_{2} \mathrm{HL}_{1}$ \\
\hline $\mathrm{LL}_{2} \mathrm{LH}_{1}$ & $\mathrm{HL}_{2} \mathrm{LH}_{1}$ & $\mathrm{LL}_{2} \mathrm{HH}_{1}$ & $\mathrm{HL}_{2} \mathrm{HH}_{1}$ \\
\hline $\mathrm{LH}_{2} \mathrm{LH}_{1}$ & $\mathrm{HH}_{2} \mathrm{LH}_{1}$ & $\mathrm{LH}_{2} \mathrm{HH}_{1}$ & $\mathrm{HH}_{2} \mathrm{HH}_{1}$ \\
\hline
\end{tabular}

Fig. (1). The DWPT for two levels.

\section{PROPOSED METHOD}

In this section, we present our proposed watermark embedding and extraction methods based on the DWPT.

\subsection{Watermark Generation}

As shown in Fig. (2), the patient information can be inserted in the electronic patient record (EPR) which includes personal information (name, age, ... etc.), clinic information (name, doctor name, ... etc.), diagnosis and some management information (record number, date, ... etc.). All the information is converted from ASCII code to binary code. After that, $\mathrm{BCH}$ error correcting code is used to encode the binary string. The resulting bitstream is the embedded watermark and using ECC enhances the robustness of the watermark.

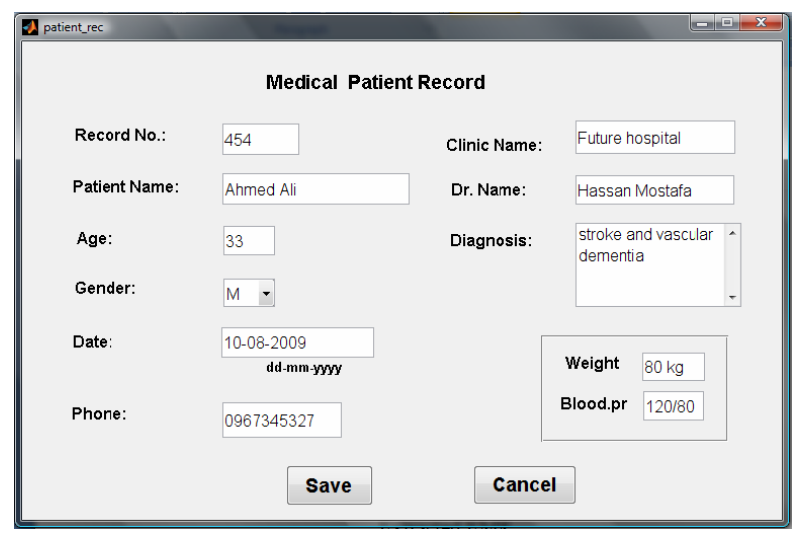

Fig. (2). Electronic patient record.

\subsection{Watermark Embedding Method}

The main steps for watermark embedding as shown in Fig. (3) are given below:

\section{Step1}

For a given $N x N$ host medical image $I$, apply $m$-level DWPT which produces $4^{m}$ sub-bands of wavelet coefficients. Each sub-band is a matrix of coefficients at a specific resolution with size $n x n$ where $n=N / 4^{m / 2}$.

\section{Step 2}

Compute the energy of each sub-band using the following equation:

$E=\frac{1}{n x n} \sum_{i=1}^{n} \sum_{j=1}^{n} c^{2}(i, j)$

where $E$ denotes the energy, $n x n$ is the size of sub-band, and $C$ is the DWPT coefficient. To ensure trade-off between robustness and imperceptibility, two sub-bands $B 1$ and $B 2$ with middle energy are selected for watermark embedding. The $B 1$ and $B 2$ are divided into $4 x 4$ blocks $b_{r, k}$ where $r$ is the sub-band number 1 or 2 and $k=1, \ldots \ldots ., n / 4$ is the block number.

\section{Step 3}

Prepare a greyscale reference image $R$ whose size is equal to $n x n$ (size of sub-band). The reference image is also divided into blocks $R_{k}$ of $4 x 4$ pixels where $k=1, \ldots, n / 4$. This reference image is used for blind embedding.

Step 4

One bit of the watermark is embedded per block. Based on the value of the bit, the block of the reference image $\boldsymbol{R}_{\boldsymbol{k}}$ is 
added to the corresponding sub-band block $b_{r, k}$ according to the following rule:

$$
b_{r, k}^{\prime}= \begin{cases}b_{r, k}+\alpha R_{k} & \text { if } w=0 \\ b_{r, k}-\alpha R_{k} & \text { if } w=1\end{cases}
$$

where $\alpha$ is the strength and $w$ is the watermark bit. The number of blocks of $B 1$ plus $B 2$ equals the number of embedded watermark bits.

\section{Step 5}

The process in step 4 is repeated up to the length of the watermark bitstream.

\section{Step 6}

Apply the inverse discrete wavelet packet transform to the modified wavelet coefficients to obtain the watermarked image $I^{\prime}$.

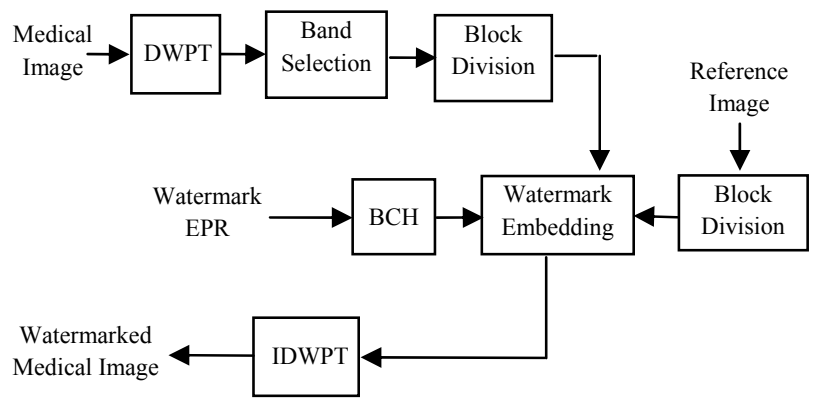

Fig. (3). Watermark embedding algorithm.

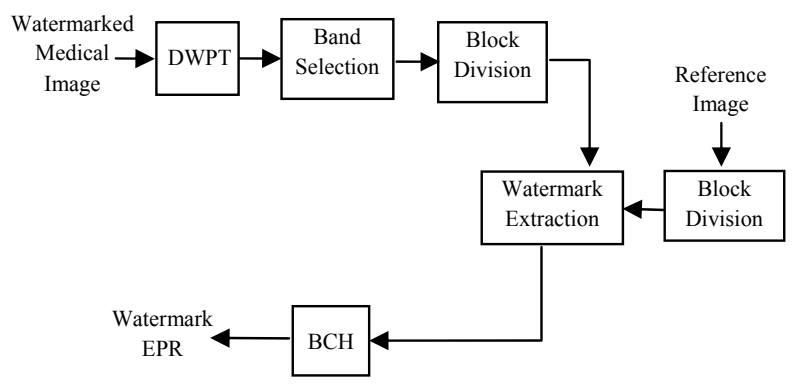

Fig. (4). Watermark extraction algorithm.

\subsection{Watermark Extraction Method}

The extraction of patient information from the watermarked medical image is blind so we don't need the original medical image to extract patient information as shown in Fig. (4). The procedure is described in the following steps:

\section{Step 1}

For a given $N x N$ watermarked (and may be attacked) medical image $I^{\prime}$, apply $m$-level DWPT which produces $4^{m}$ sub-bands of wavelet coefficients.

\section{Step 2}

Choose the same two sub-bands with middle energy used in the watermark embedding and divide them into $4 x 4$ blocks $b_{r, k}$
Step 3

Use the greyscale reference image $R$ with size $n x n$. Divide the reference image into blocks $\mathrm{R}_{k}$ of $4 \times 4$ pixels where $\mathrm{k}=1, \ldots, \mathrm{n} / 4$.

\section{Step 4}

For each block, compute the correlation coefficient value corr between the reference image block and the corresponding block of watermarked sub-bands as shown in equation 3:

$$
\text { corr }=\frac{1}{\mathbf{4} \times \mathbf{4}} R_{k} \cdot b_{r, k}
$$

The recovered watermark bit is selected as follow:

$$
w^{\prime}= \begin{cases}0 & \text { if corr } \geq 0 \\ 1 & \text { if corr }<0\end{cases}
$$

Step 5

Apply the steps of the watermark generation section in reverse order to obtain the patient information.

\section{EXPERIMENTAL RESULTS}

The performance of the proposed algorithm is tested on 8 greyscale medical images as shown in Fig. (5). Four types of medical images CT, MRI, and MRA and radiological of size $512 \times 512$ pixels have been used. By applying two level DWPT, the size of each sub-band is $128 \times 128$ pixels. Two sub-bands are chosen to embed watermark and are divided into $4 \times 4$ blocks so the total number of blocks are $2 *(128 / 4 * 128 / 4)=2048$ blocks.

The size of the patient information inserted into EPR is 136 characters which has been converted into binary matrix of 7x136 (each character represented by 7 bits). By using $\mathrm{BCH}$ code, the binary matrix becomes $15 \times 136$. The binary matrix has been converted into vector of 2040 bits. The bitstream is padded by zeros to become of length 2048 . However, each watermark bit is embedded into one block. The reference image is chosen as a hospital logo of size $128 \times 128$.

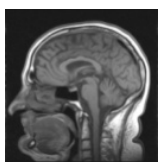

(a) MRI1

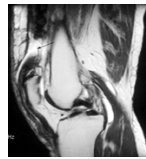

(e) MRI2

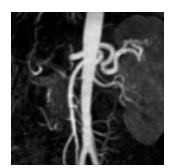

(b) MRA1

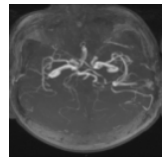

(f) MRA2

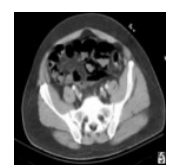

(c) CT1

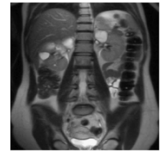

(g) CT2

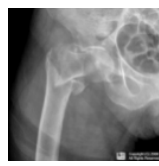

(d) Radiol

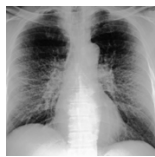

(h) Radio2
Fig. (5). Tested greyscale medical images [12].

The performance of the proposed watermarking approach is evaluated in terms of the imperceptibility and robustness against various attacks. The Peak-Signal-To-Noise Ratio (PSNR) is used to measure the visual quality of watermarked and attacked image [11] and is defined as: 
PSNR $=10 \log \frac{255^{2}}{M S E}$

where MSE is the mean squared error between the original and distorted image and is defined as follows:

MSE $=\frac{1}{m n} \sum_{i=1}^{m} \sum_{j=1}^{n}\left[l(i, j)-I^{\prime}(i, j)\right]^{2}$

where $m, n$ give the size of the image and $I(i, j), I^{\prime}(i, j)$ are the pixel values at location $(i, j)$ of the original and distorted images respectively. However, robustness is measured by the normalized correlation coefficient (NC) whose peak value is one and bit error rate (BER). The NC and BER are calculated according to the formulas (7) and (8) respectively:

$\mathrm{NC}=\frac{\sum_{i} \sum_{j} W(i, j) * W^{\prime}(i, j)}{\sqrt{\sum_{i} \sum_{j} W(i, j)^{2}} \sqrt{\sum_{i} \sum_{j} W^{\prime}(i, j)^{2}}}$

BER $=\frac{1}{h * g} \sum_{i=1}^{h} \sum_{j=1}^{g} w(i, j) \oplus w^{\prime}(i, j)$

where $h, g$ are the width and height of the watermark $w$.

Fig. (6) shows an original image MRI2 and its watermarked version. The PSNR value between these two images is $39.0999 \mathrm{~dB}$. As evidence from both images, there is no visual difference between the original and watermarked image which satisfy the strict requirements of medical image watermarking.

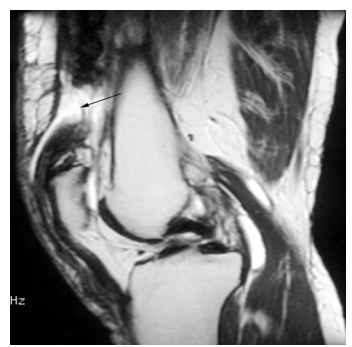

(a)

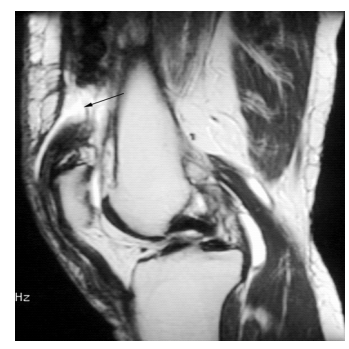

(b)
Fig. (6). (a) Original Image (b) Watermarked Image.

Table 1 shows the PSNR, NC and BER for all tested images in no attack case. The result shows that the watermark is retrieved successfully without any errors, the $\mathrm{NC}$ value is 1.000 and BER is 0.0 for all tested images.

The four types of medical images are subject to different attacks to measure the robustness of the watermarking algorithm. Tables $\mathbf{2}$ to $\mathbf{9}$, show the PSNR, NC and BER for the case of attacked images. Analysis of the results shows that our proposed scheme has high degree of robustness to sharpen and automatic equalization attacks. The patient information can be recovered without any errors, BER $=0 \%$ and the similarity between recovered and original information is equal to 1 for the four types of images as shown in Tables $\mathbf{3}$ and $\mathbf{8}$.

For Gaussian noise attack, the patient information is recovered with $\mathrm{BER}=0 \%$ and $\mathrm{NC}=1$ when the variance is
0.001 . However, with variance of 0.005 the MRI 1 and CT1images are recovered by $0.7 \%$, and $0.3 \%$ BER as shown in Table 2. For median filter attack as indicated in Table 4, the radiological image type resulted in the best robustness with $\mathrm{BER}=0 \%$ and $\mathrm{NC}=1$ while the other three types have watermark recovery errors.

Table 1. Performance of Medical Image Watermarking (no Attack)

\begin{tabular}{|c|c|c|c|}
\hline Image & PSNR & NC & BER \\
\hline \hline MRI1 & 39.0999 & 1 & 0 \\
\hline MRI2 & 39.2094 & 1 & 0 \\
\hline MRA1 & 39.3009 & 1 & 0 \\
\hline MRA2 & 39.1004 & 1 & 0 \\
\hline CT1 & 39.9446 & 1 & 0 \\
\hline CT2 & 39.0999 & 1 & 0 \\
\hline Radio1 & 39.2623 & 1 & 0 \\
\hline Radio2 & 39.1017 & 1 & 0 \\
\hline
\end{tabular}

Table 2. Gaussian Noise Attack

\begin{tabular}{|c|c|c|c|c|c|c|}
\hline \multirow{2}{*}{ Image } & \multicolumn{3}{|c|}{ Variance $=.001$} & \multicolumn{3}{c|}{ Variance $=.005$} \\
\cline { 2 - 7 } & PSNR & NC & BER & PSNR & NC & BER \\
\hline \hline MRI1 & 29.5426 & 1 & 0 & 23.1001 & 0.9919 & 0.0074 \\
\hline MRI2 & 33.3226 & 1 & 0 & 27.4583 & 1 & 0 \\
\hline MRA1 & 33.1387 & 1 & 0 & 26.7269 & 1 & 0 \\
\hline MRA2 & 32.9575 & 1 & 0 & 27.5835 & 1 & 0 \\
\hline CT1 & 32.8834 & 1 & 0 & 27.2033 & 0.9963 & 0.0032 \\
\hline CT2 & 33.4091 & 1 & 0 & 27.2776 & 1 & 0 \\
\hline Radio1 & 33.5368 & 1 & 0 & 27.8753 & 1 & 0 \\
\hline Radio2 & 34.2908 & 1 & 0 & 27.9641 & 1 & 0 \\
\hline
\end{tabular}

Table 5 illustrates the results of image attack at different values of the Gamma correction parameter (Par). The watermark can be recovered with no BER for value 2 while for value 0.5 , both CT1 and Radio1 images are recovered with $7 \%$ and $0.1 \%$ respectively. On the other side, the patient information is correctly recovered without any error in the case of contrast attack by factor 10 . But, when this factor is increased to 30 the patient information failed to be extracted from the image without error except for the case of the MRI1 and Radio 2 images as indicated by Table $\mathbf{6}$.

In Table 7 the medical image is rotated by $5^{\circ}$. Before extracting the watermark, the image is re-rotated to its original position. The results indicate that the patient information can be extracted with $\mathrm{BER}=0 \%$ and $\mathrm{NC}=1$ except for the MRI1, CT1 and CT2 images where BER= $0.2 \%, 0.4 \%$, and $0.1 \%$, and $\mathrm{NC}=0.9977,0.9951$, and 0.9988 consequently. Finally, Table 9 shows the robustness of our 
watermarking scheme against JPEG attacks with different quality factors $(\mathrm{QF})$. For $\mathrm{QF}=90 \%, 80 \%$, the BER $=0 \%$ and $\mathrm{NC}=1$ for the four types of medical images so the watermark is successfully recovered but for $\mathrm{QF}=70 \%$, and $60 \%$ the watermark is recovered with BER for some types of images.

Table 3. Sharpen Attack

\begin{tabular}{|c|c|c|c|c|c|c|}
\hline \multirow{2}{*}{ Image } & \multicolumn{3}{|c|}{ Par =.2 } & \multicolumn{3}{c|}{ Par =.6 } \\
\cline { 2 - 7 } & PSNR & NC & BER & PSNR & NC & BER \\
\hline \hline MRI1 & 24.4399 & 1 & 0 & 25.1128 & 1 & 0 \\
\hline MRI2 & 26.2089 & 1 & 0 & 27.0520 & 1 & 0 \\
\hline MRA1 & 27.0071 & 1 & 0 & 27.8567 & 1 & 0 \\
\hline MRA2 & 24.9004 & 1 & 0 & 25.6724 & 1 & 0 \\
\hline CT1 & 23.6153 & 1 & 0 & 24.3030 & 1 & 0 \\
\hline CT2 & 26.1926 & 1 & 0 & 26.9339 & 1 & 0 \\
\hline Radio1 & 24.1933 & 1 & 0 & 25.1200 & 1 & 0 \\
\hline Radio2 & 24.7326 & 1 & 0 & 25.4788 & 1 & 0 \\
\hline
\end{tabular}

Table 4. Median Filter Attack

\begin{tabular}{|c|c|c|c|}
\hline \multirow{2}{*}{ Image } & \multicolumn{3}{|c|}{$\mathbf{3 \times 3}$} \\
\cline { 2 - 4 } & PSNR & NC & BER \\
\hline \hline MRI1 & 36.1538 & 0.9736 & 0.0242 \\
\hline MRI2 & 37.3962 & 0.9645 & 0.0305 \\
\hline MRA1 & 41.1811 & 0.9902 & 0.0084 \\
\hline MRA2 & 37.7166 & 0.9915 & 0.0074 \\
\hline CT1 & 33.0626 & 0.9439 & 0.0483 \\
\hline CT2 & 27.5777 & 0.9842 & 0.0137 \\
\hline Radio1 & 32.3532 & 1 & 0 \\
\hline Radio2 & 32.7215 & 1 & 0 \\
\hline
\end{tabular}

Table 5. Gamma Correction Attack

\begin{tabular}{|c|c|c|c|c|c|c|}
\hline \multirow{2}{*}{ Image } & \multicolumn{3}{|c|}{ Par $=.5$} & \multicolumn{3}{c|}{ Par = } \\
\cline { 2 - 7 } & PSNR & NC & BER & PSNR & NC & BER \\
\hline \hline MRI1 & 14.5060 & 1 & 0 & 13.0705 & 1 & 0 \\
\hline MRI2 & 15.6436 & 1 & 0 & 15.1122 & 1 & 0 \\
\hline MRA1 & 17.6259 & 1 & 0 & 14.2096 & 1 & 0 \\
\hline MRA2 & 14.1319 & 1 & 0 & 12.5231 & 1 & 0 \\
\hline CT1 & 16.0795 & 0.9150 & 0.0714 & 15.1780 & 1 & 0 \\
\hline CT2 & 15.7198 & 1 & 0 & 13.1466 & 1 & 0 \\
\hline Radio1 & 13.4521 & 0.9988 & 0.0011 & 14.3092 & 1 & 0 \\
\hline Radio2 & 13.6493 & 1 & 0 & 14.7227 & 1 & 0 \\
\hline
\end{tabular}

Table 6. Contrast Attack

\begin{tabular}{|c|c|c|c|c|c|c|}
\hline \multirow{2}{*}{ Image } & \multicolumn{3}{|c|}{ Factor $=10$} & \multicolumn{3}{c|}{ Factor $=30$} \\
\cline { 2 - 7 } & PSNR & NC & BER & PSNR & NC & BER \\
\hline \hline MRI1 & 31.474 & 1 & 0 & 22.471 & 1 & 0 \\
\hline MRI2 & 31.454 & 1 & 0 & 22.347 & 0.982 & 0.016 \\
\hline MRA1 & 30.586 & 1 & 0 & 22.308 & 0.979 & 0.018 \\
\hline MRA2 & 31.814 & 1 & 0 & 23.343 & 0.995 & 0.004 \\
\hline CT1 & 34.351 & 1 & 0 & 25.079 & 0.888 & 0.092 \\
\hline CT2 & 30.787 & 1 & 0 & 22.286 & 0.996 & 0.003 \\
\hline Radio1 & 34.449 & 1 & 0 & 25.680 & 0.995 & 0.004 \\
\hline Radio2 & 33.120 & 1 & 0 & 24.524 & 1 & 0 \\
\hline
\end{tabular}

Table 7. Rotate Attack

\begin{tabular}{|c|c|c|c|}
\hline \multirow{2}{*}{ Image } & \multicolumn{3}{|c|}{ Rotation $\left(\mathbf{5}^{\mathbf{0}} \rightarrow \mathbf{- 5}^{\mathbf{0}}\right)$} \\
\cline { 2 - 4 } & PSNR & NC & BER \\
\hline \hline MRI1 & 29.8776 & 0.9977 & 0.0021 \\
\hline MRI2 & 23.3739 & 1 & 0 \\
\hline MRA1 & 32.2934 & 1 & 0 \\
\hline MRA2 & 30.5850 & 1 & 0 \\
\hline CT1 & 28.0750 & 0.9951 & 0.0042 \\
\hline CT2 & 21.5120 & 0.9988 & 0.0011 \\
\hline Radio1 & 21.2127 & 1 & 0 \\
\hline Radio2 & 18.0939 & 1 & 0 \\
\hline
\end{tabular}

Table 8. Automatic Equalization Attack

\begin{tabular}{|c|c|c|c|}
\hline Image & PSNR & NC & BER \\
\hline \hline MRI1 & 13.0778 & 1 & 0 \\
\hline MRI2 & 26.5190 & 1 & 0 \\
\hline MRA1 & 10.1132 & 1 & 0 \\
\hline MRA2 & 10.7452 & 1 & 0 \\
\hline CT1 & 18.1084 & 1 & 0 \\
\hline CT2 & 10.2840 & 1 & 0 \\
\hline Radio1 & 19.4670 & 1 & 0 \\
\hline Radio2 & 20.2793 & 1 & \\
\hline
\end{tabular}

\section{CONCLUSIONS}

In this paper, a new blind watermarking scheme has been introduced and developed to embed patient information in a private and secure manner. The proposed scheme satisfies the security of medical patient information and allows 
Table 9. JPEG Compression Attack

\begin{tabular}{|c|c|c|c|c|c|c|c|c|c|c|c|c|}
\hline \multirow{2}{*}{ Image } & \multicolumn{3}{|c|}{$Q F=90 \%$} & \multicolumn{3}{|c|}{$\mathrm{QF}=\mathbf{8 0} \%$} & \multicolumn{3}{|c|}{$\mathrm{QF}=70 \%$} & \multicolumn{3}{|c|}{$\mathrm{QF}=60 \%$} \\
\hline & PSNR & $\mathrm{NC}$ & BER & PSNR & $\mathrm{NC}$ & BER & PSNR & NC & BER & PSNR & NC & BER \\
\hline MRI1 & 39.1283 & 1 & 0 & 39.1954 & 1 & 0 & 38.7686 & 1 & 0 & 39.2142 & 0.9606 & 0.0357 \\
\hline MRI2 & 38.7412 & 1 & 0 & 38.6044 & 1 & 0 & 37.8652 & 1 & 0 & 38.4243 & 0.9890 & 0.0095 \\
\hline MRA1 & 39.3926 & 1 & 0 & 39.4710 & 1 & 0 & 39.3432 & 1 & 0 & 40.4580 & 0.8575 & 0.1229 \\
\hline MRA2 & 38.3999 & 1 & 0 & 38.0280 & 1 & 0 & 37.3049 & 1 & 0 & 37.2851 & 1 & 0 \\
\hline CT1 & 38.8473 & 1 & 0 & 37.8439 & 1 & 0 & 36.9995 & 0.9667 & 0.0284 & 36.0498 & 0.9590 & 0.0347 \\
\hline CT2 & 38.9885 & 1 & 0 & 38.9252 & 1 & 0 & 38.0778 & 1 & 0 & 39.2624 & 0.9939 & 0.0053 \\
\hline Radio1 & 37.6862 & 1 & 0 & 37.2173 & 1 & 0 & 36.5652 & 1 & 0 & 36.1547 & 1 & 0 \\
\hline Radio2 & 38.2032 & 1 & 0 & 38.0067 & 1 & 0 & 37.3545 & 1 & 0 & 37.4947 & 1 & 0 \\
\hline
\end{tabular}

sharing the medical information remotely and manageably without extra cost or storage space and without any effect on medical image quality. We examined the security of the scheme by applying some attacks and measuring the robustness of the scheme by BER and NC and examining the visual quality of medical image by PSNR.

Experimental results show that the proposed scheme is robust against common attacks such as Gaussian noise, gamma correction, automatic equalization, contrast adjustment, rotation, and sharpening, median filter and JPEG compression with different QF. Also it has been noticed that the Radiological image is the most robust type of medical image against attacks. Although the scheme resists most attacks, there is some none satisfactory performance in the area of median filtering and contrast adjustment by factor 30 .

In the future research, we will try to enhance our algorithm in order to obtain watermarked medical images with less degradation and to have recovered watermark with better accuracy.

\section{ACKNOWLEDGEMENT}

The authors would like to acknowledge the Encyclopedia of medical images as the source of the images used in this research (http://www.images.md/).

\section{REFERENCES}

[1] G. Kontazakis, "Telemedicine: Current technological status, applications and future aspects", In: Proc. Biomedical Imaging IV Lecturers and Participants, 4th IEEE-EMBS International Summer School on Biomedical Imaging, France, June 2000.

[2] D. Ananad, and U.C. Niranjan, "Watermarking medical images with patient information", In: proc. IEEE/EMBS Conference, Hong Kong, China, Oct 1998, pp. 703-706.
[3] J. Nayak, P.S. Bhat, M.S. Kumar, and R. Acharya, "Reliable transmission and storage of medical images with patient information using error control codes", In: Proc. IEEE INDICON, pp.147-150, 2004.

[4] M. S. Nambakhsh, A. Ahmadian, M. Ghavami, R. S. Dilmaghani, and S. Karimi-Fard, "A Novel Blind Watermarking of ECG Signals on Medical Images using EZW Algorithm", 28th Annual International Conference of the IEEE Engineering in Medicine and Biology Society, pp. 3274-3277, Aug. 2006.

[5] A. Giakoumaki, S. Pavlopoulos, and D. Koutsouris, "Multiple image watermarking applied to health information management", IEEE Trans. Inf. Technol. Biomed., vol. 10, no. 4, pp. 722-732, Oct. 2006.

[6] M. Kallel, J. Lapayre, and M.S. Bouhlel, "A multiple watermarking scheme for medical image in the spatial domain", GVIP J., vol. 7, no. 1, pp. 37-42, 2007.

[7] H. C. Huang, W. C. Fang, S. C. Chen, "Privacy Protection and Authentication for Medical Images with Record-Based Watermarking", in IEEE/NIH LIfe Science Systems and Applications Workshop, Bethesda, MD, USA, 2009.

[8] R. R. Colín, C. F. Uribe, and J. A. M. Villanueva, "Robust watermarking scheme applied to radiological medical images", IEICE Trans. Inf. Syst. vol. E91-D, no. 3, pp. 862-864, 2008.

[9] W. Dietl, and A. Uhl, "Watermark security via secret wavelet packet subband structures", In: A. Lioy, and D. Mazzocchi, Eds., Communications and Multimedia Security. Proceedings of the Seventh IFIP TC-6 TC-11 Conference on Communications and Multimedia Security, Turin, Italy, Lecture Notes on Computer Science, vol. 2828, pp. 214-225, Oct. 2003.

[10] P. Kumhom, S. On-rit, K. Chamnongthai, "Image watermarking based on wavelet packet transform with best tree", ECTI Trans. Electr. Eng. Electron. Commun., vol. 2, no.1, pp. 23-35, February 2004.

[11] B. Plaintz and A. Maeder, "Medical image watermarking: A study on image degradation", Proc. of the Australian Pattern Recognition Society (APRS) Workshop on Digital Image Computing (WDIC 2005), pp. 3-8, 2005.

[12] The Online Encyclopaedia of Medical Images, http://www.images.md/

(C) Mostafa et al.; Licensee Bentham Open.

This is an open access article licensed under the terms of the Creative Commons Attribution Non-Commercial License (http://creativecommons.org/licenses/by-nc/3.0/) which permits unrestricted, non-commercial use, distribution and reproduction in any medium, provided the work is properly cited. 